\title{
THE $\mu$ HAMMER: \\ INVESTIGATING CELLULAR RESPONSE TO IMPACT WITH A HIGH THROUGHPUT MICROFLUIDIC MEMS DEVICE
}

\author{
Luke H.C. Patterson ${ }^{1}$, Jennifer L. Walker ${ }^{1}$,Evelyn Rodriguez-Mesa', Kevin Shields ${ }^{2}$, John S. Foster ${ }^{2}$, \\ Megan T. Valentine ${ }^{1,3,4}$, Adele M. Doyle ${ }^{3,4}$, and Kimberly L. Foster ${ }^{1,4}$ \\ ${ }^{1}$ Department of Mechanical Engineering, ${ }^{3}$ Neuroscience Research Institute, and ${ }^{4}$ Center for Bioengineering, \\ University of California, Santa Barbara, California, USA \\ ${ }^{2}$ Owl Biomedical, Goleta, California, USA
}

\begin{abstract}
We report the application of stress to biological cells at unprecedented strain $(50 \%)$, strain rate $\left(180,000 \mathrm{~s}^{-1}\right)$, and throughput (1,800 cells/min) using a high-speed, high actuation force magnetically-driven MEMS chip. This device is uniquely suited to study the effects of impact on large populations of inherently heterogeneous cells, enabling statistical analysis that can elucidate the cell-level ramifications of Traumatic Brain Injury (TBI). To demonstrate the capabilities of the $\mu$ Hammer, we applied TBIrelevant strains and strain rates to human leukemic K562 cells then monitored their proliferation for 9 days. We observed significantly repressed proliferation of the hit cells compared to both the negative and sham controls, indicating success in applying sublethal cellular damage.
\end{abstract}

\section{INTRODUCTION}

According to the CDC, approximately 2 million Traumatic Brain Injuries (TBIs) occur in the United States each year, resulting from either physical impacts such as those common in sports injuries or percussive waves such as those following explosive blasts [1]. The symptoms of these injuries can linger for years after the incident and have far-reaching medical, societal, and economic impact. Little is known about TBI's mechanisms of injury and loss of function, however, especially on the cellular level. This is due in part to the lack of instruments to replicate TBI-relevant loading conditions on individual cells. While several device platforms capable of applying compressive forces to individual cells exist (e.g. AFM, microplates), each is limited to some combination of low force $($ sub- $\mu \mathrm{N})$, strain $(<10 \%)$, and strain rate $\left(<10 \mathrm{~s}^{-1}\right)$ [2-4]. Even more limiting is the low throughput of these devices (tens of cells per experiment), which makes gathering sufficient data for sensitive biological analysis of inherently heterogeneous cell populations difficult if not impossible [5]. These deficiencies have particularly hindered investigations of TBI, which typically involve peak tissuelevel strains of $10-50 \%$ and strain rates of $10-1000 \mathrm{~s}^{-1}$, parameters that may be even higher on the cellular level due to localized heterogeneities $[6,7]$.

To address these critical needs, we have developed a microfluidic MEMS device, the " $\mu$ Hammer," to subject individual cells to impact with an unprecedented combination of strain (10$75 \%$ ), strain rate $\left(10,000+\mathrm{s}^{-1}\right)$, and throughput (up to 3,000 cells/min). Cells are processed under sterile conditions and can be removed after the loading event for immediate analysis or cultured in vitro for long term tracking. Here, we demonstrate the capabilities of the $\mu$ Hammer by applying TBI-relevant strains and strain rates to a prototypical cell line, human leukemic K562 cells, and then monitoring their proliferation for 9 days.

\section{MATERIALS AND METHODS \\ $\mu$ Hammer Components}

The $\mu$ Hammer is composed of single-crystal silicon with an embedded $\mathrm{NiFe}$ armature in microchip format, magnetically driven by an external solenoid via an in situ NiFe pole (Figure 1). The device is fabricated by Owl Biomedical (USA) using a 14-layer process, with the silicon features etched via RIE/DRIE and the $\mathrm{NiFe}$ features deposited via electrochemical plating. The upper surface of the device is sealed with glass, while the bottom surface is bonded to a macroscale interposer that interrogates the three vias (one input, one output, one waste). This is finally bonded to a sterile cartridge, which holds up to $10 \mathrm{~mL}$ of input cells suspended in buffer and
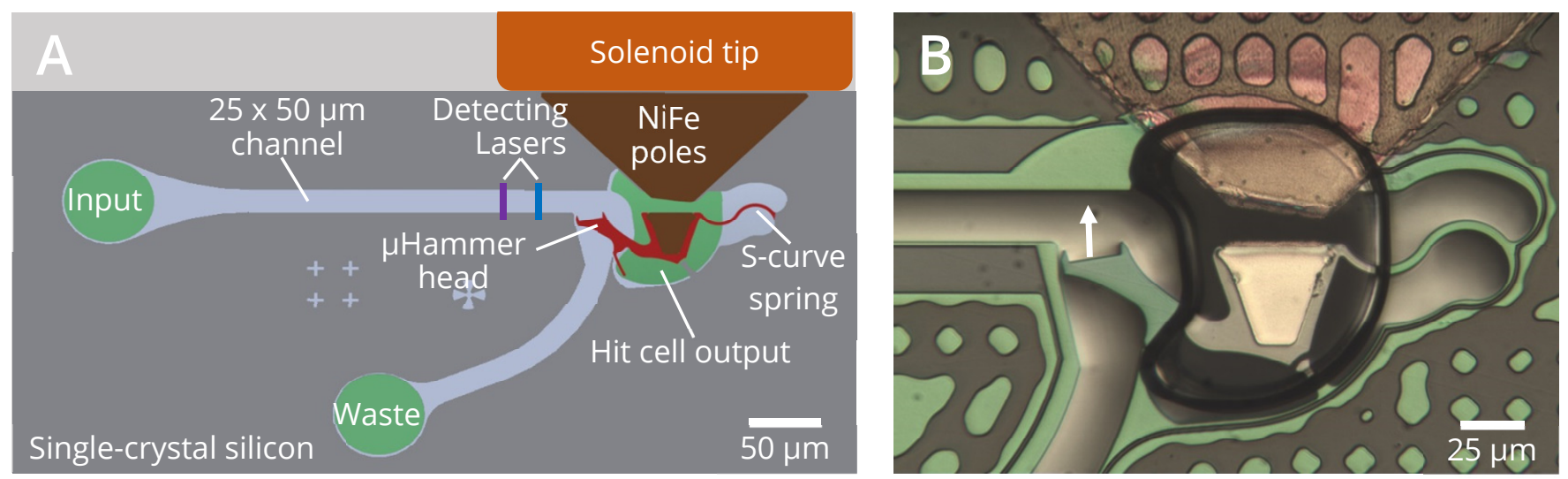

Figure 1A: $\mu$ Hammer device schematic. Chip (dark grey) is bonded to a sterilized macroscale cartridge (not shown) which interfaces with a flow-control pump and can be loaded with cells suspended in buffer. Cells enter chip through the input via, flow through channel, and are impacted by $\mu$ Hammer when external solenoid is activated, swinging lower magnet and $\mu$ Hammer head upward. Impacted cells exit through output via and are extracted for analysis. Figure 1B: $\mu$ Hammer micrograph. Arrow indicates direction of $\mu$ Hammer actuation. 

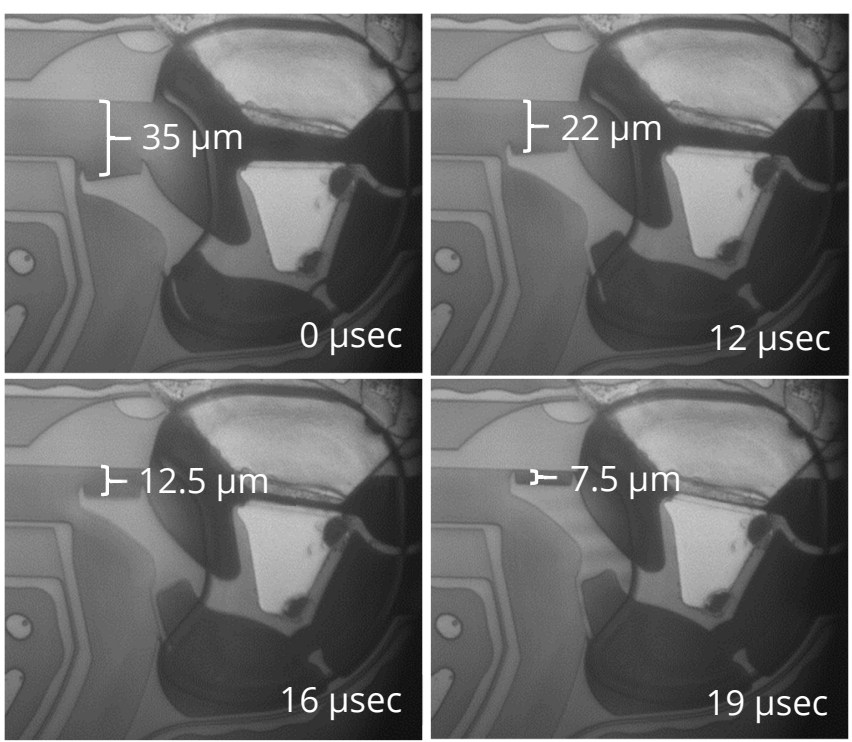

Figure 2: Strobed images of $\mu$ Hammer actuation without cells. Images taken at $1 \mu$ intervals after solenoid trigger. Hundreds of images are averaged at each timestep to create the final image. Image tracking software is then used to track the gap distance (see white brackets) as a function of time.

collects the output for immediate extraction and analysis. The cartridge interfaces with the MACSQuant ${ }^{\circledR}$ Tyto ${ }^{\circledR}$ (Miltenyi Biotech, Germany) system with a flow-controlled pump. This pump applies positive pressure $(120 \pm 20$ mbar $)$ to flow fluorescently labeled cells through the input via to the device, where they pass through a $25 \mu \mathrm{m} \times 50 \mu \mathrm{m}$ channel at a controlled velocity $(1.25 \pm$ $0.3 \mathrm{~m} / \mathrm{s})$. Two lasers aligned with the channel detect fluorescently labeled cells, both to monitor the speed for timing and to trigger the solenoid at a predetermined interval before the cell will reach the $\mu$ Hammer head (the 'pre-arrival time'). The released $\mu$ Hammer head extends from the $\mathrm{NiFe}$ armature and is anchored to the surrounding silicon substrate by a released S-curve spring. Once actuated, the $\mu$ Hammer head rotates about the anchor point at a tunable speed (that varies with current applied to the solenoid) across the channel to full actuation, where it can be held for a userspecified period before returning to rest to prepare for the next hit.

\section{Device Characterization}

To characterize the stiffness of the S-curve spring, we created a two-dimensional steady-state finite element model of the $\mu$ Hammer in COMSOL, solving for structural deformation under the influence of a point load on the edge of the released $\mathrm{NiFe}$ armature. The magnitude of this force was determined by a separate finite-element model of the solenoid and $\mathrm{NiFe}$ poles, solving for both the electric and magnetic fields as well as the resulting electromagnetic force.

To characterize the velocity of actuation and the subsequent strain rate the device applies, we used a high-speed camera to capture strobed images of the device at $1 \mu$ sintervals after triggering the solenoid. We then used feature-tracking software to determine the gap distance between the $\mu$ Hammer face and the top of the channel as a function of time (Figure 2). We did this first with buffer flowing through the channel and no cells present to determine the actuation profile of the valve and thus to select an appropriate prearrival time. We then used this information to successfully impact cells flowing through the channel, and captured strobed images of the $\mu$ Hammer under these conditions as well.

To validate this pre-arrival time for our system, we updated our COMSOL model of the $\mu$ Hammer to be time-dependent, solving for structural deformation, fluid velocity, and particle (cell) position. In this model, a fluid pressure was applied at the inlet of the device, which carried a $15 \mu \mathrm{m}$ diameter model cell through the channel while the previously determined boundary magnetic force was applied to the $\mathrm{NiFe}$ armature for actuation.

\section{K562 Impact Study}

Human K562 cells (ATCC, USA) were fluorescently labeled with carboxyfluorescein succinimidyl ester (CFSE, Thermo Fisher Scientific, USA) which has excitation/emission of $492 / 517 \mathrm{~nm}$. The cells were then suspended at 50,000 cells $/ \mathrm{mL}$ in MACSQuant ${ }^{\circledR}$ Tyto ${ }^{\circledR}$ Running Buffer (Miltenyi Biotech, Germany), and flowed through the $\mu$ Hammer's sterilized microfluidic channel at 100,000 cells/hour. As each cell passed through the lasers, it was detected and timed for a $\mu$ Hammer impact which subjected each cell to $50 \%$ strain at a $180,000 \mathrm{~s}^{-1}$ strain rate (see Figure 3 for impact profile). A sham control population was fluorescently stained and flowed through the device without impact, while a negative control population was neither fluorescently stained nor introduced into the device.

After each treatment, four replicates of each group were seeded at 40,000 cells $/ \mathrm{mL}$ and cultured in 12-well cell culture microplates (Thermo Fisher Scientific, USA). K562 cells were grown in RPMI1640 media supplemented with 10\% FBS, $25 \mathrm{mM}$ HEPES, and $0.01 \%$ penicillin streptomycin (Lonza Biologics, UK) at $37^{\circ} \mathrm{C}$ and $5 \% \mathrm{CO} 2$. Every 24 hours, cell density was measured in triplicate by flow cytometry (MACSQuant ${ }^{\circledR}$ Flow Cytometer, Miltenyi Biotech, Germany) to monitor each group's proliferation rate until the cell number reached $1,000,000$ cells/well. After the density measurement on day 7 , fresh media was added to the hit population wells to maintain sufficient volume for continued culturing. The doubling time, $t_{\text {double }}$, of each cell population's exponential growth phase was determined by plotting the number of cells, $N$, as a function of time

where

$$
N=N_{o} \mathrm{e}^{r t}
$$

$$
t_{\text {double }}=\frac{\ln (2)}{r} .
$$

Significant difference between groups was determined via One-Way ANOVA with post-hoc Tukey's.

\section{RESULTS}

\section{Device Characterization}

As determined by observing the deflection of the $\mathrm{NiFe}$ armature in response to a point load, the stiffness of the S-curve spring is $85 \pm 5 \mathrm{~N} / \mathrm{m}$, based on a Young's modulus for silicon of 170 $\pm 10 \mathrm{GPa}$. The magnitude of the magnetic force applied to the released $\mathrm{NiFe}$ armature by the solenoid at saturation increases from 0.29 to $1.2 \mathrm{mN}$ as the armature travels toward the NiFe pole above. When this force is applied to the armature, it is pulled upward toward the top of the channel along with the $\mu$ Hammer head until it contacts the stationary NiFe pole above approximately $19 \mu$ s later, as shown in Figures 2 and 3. During transit, the $\mu$ Hammer head travels at an average speed of $2.7 \pm 0.2 \mathrm{~m} / \mathrm{s}$ during compression while closing to a $7.5 \mu \mathrm{m}$ final gap (Figure 3). This translates to a strain rate of $180,000 \pm 13,000 \mathrm{~s}^{-1}$ and $50 \%$ maximum strain for a 


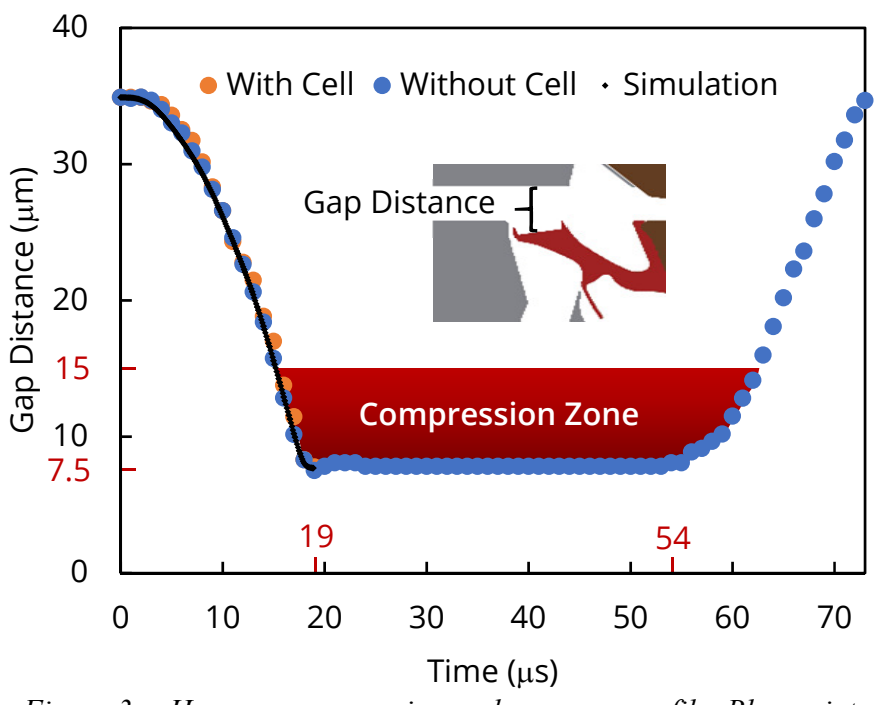

Figure 3: $\mu$ Hammer compression and recovery profile. Blue points from strobed images of devices actuated without cells. Images taken at $1 \mu$ intervals after solenoid activation until full recovery after release (full actuation held for $35 \mu$ sefore release). $n=4$ devices, standard deviation less than marker size. Orange points from strobed images while impacting cells up to full actuation $(n=2$ devices). Black line obtained using COMSOL simulations up to full actuation. Slope of the least squares best fit line in compression zone without cells is $2.7 \pm 0.2 \mathrm{~m} / \mathrm{s}$, while slope with cells is $2.8 \pm 0.2 \mathrm{~m} / \mathrm{s}$. Red marks on y-axis denote bounds of compression zone, while red marks on $x$-axis denote times of full actuation and release.

typical $15 \mu \mathrm{m}$ diameter K562 cell. Once the $\mu$ Hammer is released, it returns to rest and is ready for the next actuation within $25 \mu \mathrm{s}$.

Due to the depth of the channel $(50 \mu \mathrm{m})$, the majority of cells pass through the channel at a depth outside the focal plane of the camera. Combined with the large number of images averaged together to create each timestep, this currently limits our ability to resolve individual cells during impact. However, we were able to track the actuation profile of the $\mu$ Hammer head while impacting cells, which is almost identical to the actuation profile of the $\mu$ Hammer without cells in buffer (Figure 3). Furthermore, the hammer head velocity in the compression zone with cells $(2.8 \pm 0.2$ $\mathrm{m} / \mathrm{s}$ ) is within the reported range of hammer head velocities without cells, indicating that the cell's presence does not alter the actuation of the $\mu$ Hammer. Since the Young's modulus of a cell (on the order of $\mathrm{kPa}$ ) is orders of magnitude lower than the Young's modulus of silicon $(170 \mathrm{GPa})$, this is not surprising and indicates that no detectable strain stiffening of the cell is occurring [8].
As shown in Figure 3, there is close agreement between the experimental actuation profiles and the simulated profile from our time-dependent COMSOL model of fluid-structure interaction. This model was run with the timing parameters calculated in the previous section, resulting in the plots shown in Figure 4. In this model, our simulated cell successfully flows through the channel with a smooth trajectory until impacting the middle of the $\mu$ Hammer face, demonstrating the correct parameters were used to time the solenoid actuation and subsequent cell impact.

\section{K562 Impact Study}

Hit, sham, and negative control cells were seeded for continued culture and measurement of cell proliferation. Although the three experimental groups were seeded at the same concentration of 40,000 cells $/ \mathrm{mL}$ (for a total of 60,000 cells/well), the hit population's proliferation appears depressed compared to the sham and negative control populations (Figure 5). Both the sham and negative control populations grew exponentially from day 1 through day 6, at which point both groups surpassed 1,000,000 cells/well. During this exponential period, they both had a doubling time of approximately 27 hours. The hit cell population, however, did not enter exponential growth until day 2 (at least 24 hours later than the sham and negative controls), after which it had a doubling time of approximately 32 hours to reach 1,000,000 cells/well by day 9 .

The lack of significant difference between the negative and sham controls demonstrates the extended lag phase and increased doubling time of the hit population is the result of the $\mu$ Hammer impact alone and not other variables arising from the device microfluidics. Further, the fact that the hit cells retained their ability to proliferate post-impact demonstrates that basic cellular functions (e.g. cell division and metabolism) of the population were not irreparably compromised following impact. This indicates the $\mu$ Hammer was not only successful in applying sublethal cellular damage, which is necessary for performing biological analyses of impacted cells on longer timescales, but also may recapitulate aspects of TBI-like damage.

\section{CONCLUSIONS}

Using K562 cells as proof-of-concept, we demonstrated the $\mu$ Hammer's ability to apply consistent high strain and strain rate impacts to large populations of cells. We further showed that these impacts repressed subsequent proliferation without compromising overall viability. Such controlled loading profiles can be applied to any suspended cell or microscale material (maximum diameter $<20$ $\mu \mathrm{m})$, opening doors for microscale materials characterization at high

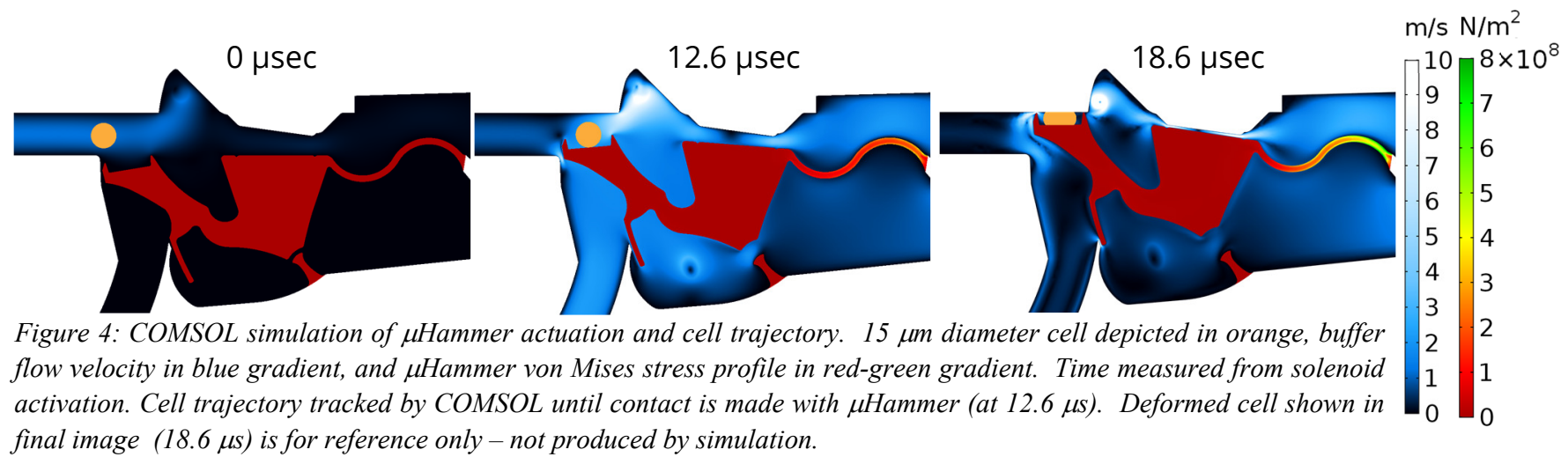




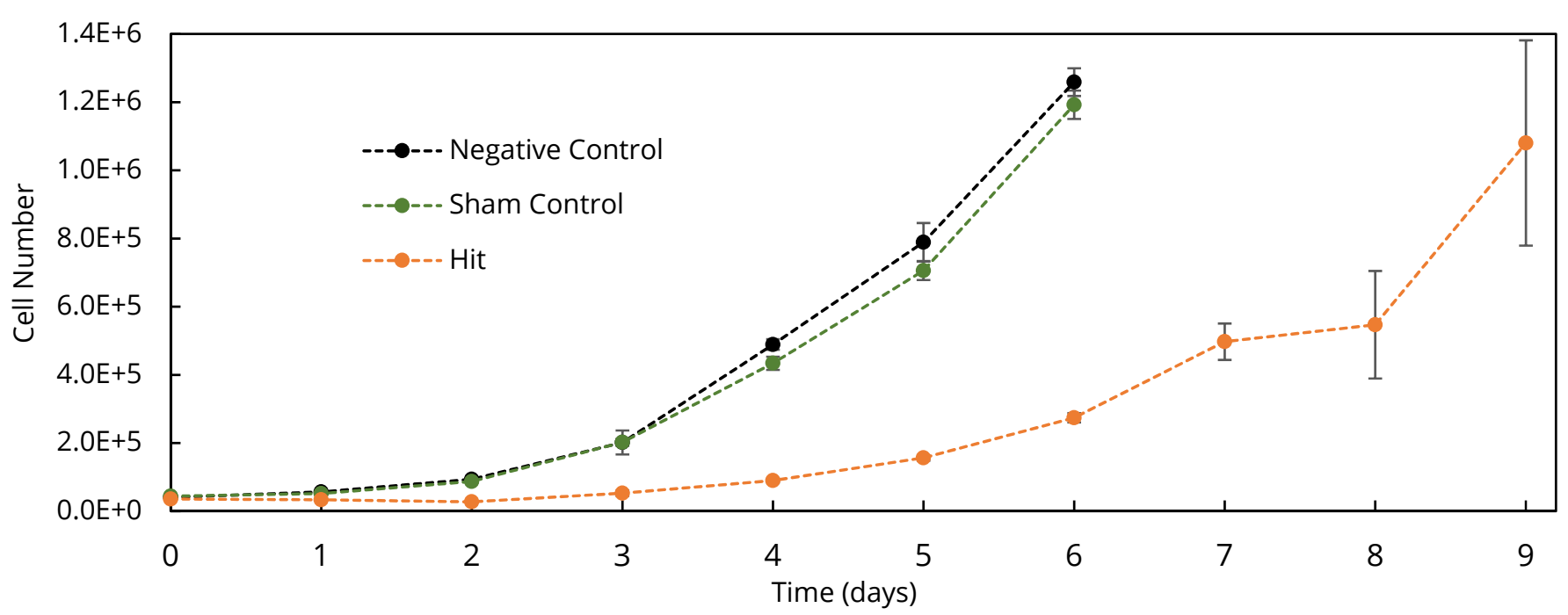

Figure 5: K562 cell growth over 9 days culturing post- $\mu$ Hammer experiment. Hit cell strain rate was 180,000 $\mathrm{s}^{-1}$, total strain 50\%, held for $35 \mu$ s (see Figure 3 for loading profile). All groups seeded at 60,000 total cells (at a density of 40,000 cells $/ \mathrm{mL}$ ) on day $0 . n=4$ wells per group, all taken from same initial population of cells. Error bars depict standard deviation. At each time point there is no significant difference between the negative and sham controls.

strain rates or investigations of force-induced trauma in a number of biological systems.

Going forward, we are particularly interested in extending our analysis to neural and other brain injury-related cells. Though the forces and various extracellular cues applied by the $\mu$ Hammer to a single cell in suspension are likely different than those experienced by a cell embedded in brain tissue in vivo, the information provided by the $\mu$ Hammer provides a simplified model system to help fill the gap in our understanding of the relationship between force and injury in cells. Due to the $\mu$ Hammer's unprecedented combination of well-defined impact parameters and high throughput, we believe it is uniquely suited to study TBI on the cellular level. As such, our next step is to perform the same proliferation study with brainderived cells to determine if they also exhibit repressed proliferation following impact, and if so, to determine the underlying reasons for this response. Future work can explore the broader range of functional, damage, and stress-related cellular assays at various strains and strain rates, investigating the link between these parameters and cellular injury. Through these and further studies, we hope to elucidate the mechanisms of cellular damage and recovery in the brain, ultimately leading to an improved understanding of impact-based cellular injuries and their treatment.

\section{ACKNOWLEDGMENTS}

Authors acknowledge support from the National Science Foundation through awards CBET-1631656 (to KLF, AMD and MTV) and CMMI-1254893 (to MTV). We also thank Carl Meinhart from the Department of Mechanical Engineering, UCSB, whose time-dependent COMSOL fluid-structure interaction model formed the basis of our own.

\section{REFERENCES}

[1] Centers for Disease Control and Prevention, "Rates of TBIrelated Emergency Department Visits, Hospitalizations, and Deaths - United States, 2001 - 2010”, https://www.cdc.gov/ traumaticbraininjury/data/rates.html (2016).

[2] M. P. Stewart, Y. Toyoda, A. A. Hyman, D. J. Muller, "Tracking mechanics and volume of globular cells with atomic force microscopy using a constant-height clamp", Nature Protocols, 7, 143-154 (2012).

[3] B. P. Chan, C. H. Li, K. L. Au-Yeung, K. Y. Sze, A. H. Ngan, "A microplate compression method for elastic modulus measurement of soft and viscoelastic collagen microspheres", Annals of Biomedical Engineering, 36, 1254-1267 (2008).

[4] K. C. Neuman, A. Nagy, "Single-molecule force spectroscopy: optical tweezers, magnetic tweezers and atomic force microscopy", Nature Methods, 5, 491-505 (2008).

[5] D. Desmaele, M. Boukallel, S. Regnier, "Actuation means for the mechanical stimulation of living cells via microelectromechanical systems: A critical review", Journal of Biomechanics, 44, 1433-1446 (2011).

[6] D. F. Meaney, B. Morrison, C. D. Bass, "The mechanics of traumatic brain injury: a review of what we know and what we need to know for reducing its societal burden", Journal of Biomechanical Engineering, 136, 021008 (2014).

[7] F. Pervin, W. W. Chen, "Dynamic mechanical response of bovine gray matter and white matter brain tissues under compression", Journal of Biomechanics, 42, 731-735 (2009).

[8] N. Guz, M. Dokukin, V. Kalaparthi, I. Sokolov, "If Cell Mechanics Can Be Described by Elastic Modulus: Study of Different Models and Probes Used in Indentation Experiments", Biophysical Journal, 107.3, 564, (2014)

\section{CONTACT}

*L.H.C. Patterson, tel: +1-760-258-6341; lpatterson@ucsb.edu 